\title{
Bankart Lesion: Comparison between Open and Arthroscopic Techniques
}

\author{
Osvandré L*, Paulo P, Carlos C, Alaor BN and Cristiano G \\ Orthopedic Surgery, Brazil
}

*Corresponding author: Osvandré L, Orthopedic Surgery, Brazil

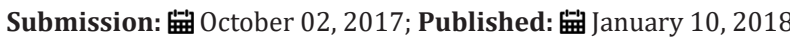

\begin{abstract}
Purpose: To compare the results from open and arthroscopic Bankart lesion repair.

Methods: Retrospective study of clinical records review and telephone contact of results after one year follow up of Bankart lesion surgery in a total of 56patients, 38 with arthroscopic repair and 18 with open repair. We evaluated the epidemiology as mean and frequency, the results were compared with instability Carter - Rowe score and submitted to independent analysis groups.
\end{abstract}

Results: Prevalence of Bankart lesions was higher in men (87\%), dominant side (59\%) and more frequent in patients less than 30 years (73.2\%). There was surgical failure in $5.3 \%$ of cases. There was no statistical significative difference result in Carter - Rowe score. ROM results were better in arthroscopic repair group. Conclusion: Both techniques have good functionality results, with a better gain of mobility for the arthroscopic technique but with an increase rate of recurrence than the open repair.

Conclusion: (1) Both the techniques have good results, even though few studies in the literature comparing homogeneous groups of Patients (2) The possibility of further episodes of dislocation is apparently greater in the arthroscopic technique (3) The arthroscopic technique seems to have a better result when compared to mobility (4) It is important to be able to correctly indicate the surgery and correctly perform the adopted technique.

\section{Introduction}

The study of shoulder instability is describe in detail since Hippocrates. The anatomy, mechanisms and different forms of treatment come to the generations being modified and perfected. The gleno humeral joint is the most mobile of the human body and is thus the most susceptible to dislocations, with an incidence of 17 cases per 100,000 inhabitants per year, with the previous instability accounting for about $85 \%$ of the cases $[1,2]$.

The congruence of this complex joint is maintained by static stabilizers: bone compliance, glenoid lip, adhesion cohesion effect, joint capsule, glenohumeral and coracoumeral ligaments. Dynamic stabilizers: cuff rotator cuff (subscapular, supraspinatus, infraespinal and minor round), scapular muscles (anterior serratus, trapezius, latus of the dorsum, rhomboids and lift of the scapula) and long biceps [3]. The importance of the stability achieved by the anterior lower edge of the glenoid lip was first described in 1906 by Phertes [4]. In 1939Bankart describes for medical community its surgical technique based on the open repair of the glenohumeral dislocation [5].

Surgical Treatment is indicate in cases of post-traumatic glenoumeral instability with open or arthroscopic technique. We specifically in cases has glenolabial injury, Hill-Sachs $<25 \%$ and anteroposterior impairment Gives long Gives lower surface over one third of the overall diameter glenoidque [6,7]. The objective of this study is to evaluate the epidemiology and the postoperative results obtained after one year comparing these two techniques, open and arthroscopic.

\section{Material and Methods}

A retrospective study of 66 patients submitted to Bankart's surgery between January 2009 and December 2013 at the Rio Grande do Sul Institute of Orthopedics and Traumatology (IOTRS). Of these, 20 were operated by open technique and 46 by arthroscopic technique. Epidemiological aspects were evaluated through the analysis of medical records and telephone contact. The results obtained after one year of postoperative were analyzed using the functional score criteria for Carter-Rowe shoulder instability. For the comparison methodology, the study was done for independent groups.

Inclusion criteria:

a. Patients with relapsed anterior traumatic instability.

b. When present, glenoid bone lesions or Hill Sacks $<25 \%$.

c. Primary lesion of the glenoid lip.

The following were excluded:

a. Those who did not follow the rehabilitation protocol as directed. 
b. Did not give outpatient segments at least 1 year post operative.

c. Incomplete medical records.

d. Surgical failures that necessitated reintervention due to poor positioning of the anchors.

After the inclusion criteria exclusion stage, 56 patients remained. Of these, 38 were operated by the arthroscopic technique and 18 by the open technique. All patients were operated by members of the Brazilian Society of Shoulder and Elbow Surgery. The choice of the surgical technique and the type of material used was according to the surgeon's choice and taking into account the release of the material by the agreements or budgetary possibilities when the surgeries were private.

Arthroscopic technique is performed by general anesthesia associated with brachial plexus block. Patient in the beach chair position and the operated limb was submitted to traction as required in the intraoperative period. Posterior portal for intraarticular visualization with saline solution infusion. Bankart lesion is identified and debridement of the glenoid bone border and release of the medialized capsule and labrum is realized. We use multiple bio-absorbable anchors according to the extent of the lesion for capsular-labral repair. Centering of the humeral head on the glenoid must be observe after complete de repair, demonstrating good positioning. Skin incisions are close with 4-0 nylon.

Open technique is performed by general anesthesia too, associated with brachial plexus block. Deltopectoral approach is preferred. Tenotomy of the subscapular tendon is performed $1 \mathrm{~cm}$ medial to the lesser tuberosity along with the joint capsule. Identification of the Bankart lesion, debridement of the glenoid bone and release of the medialized capsule and labrum were performed. Suture metallic anchors were used for labral reconstruction and capsular shift retencionament. Subscapular tendon repair is performed with braided non-absorbable suture. At the end performed hemostasis and suture by plans up to the skin.

Rehabilitation protocol:

a. Immobilization with sling for 3-6 weeks, depending on the size of the lesion.

b. Initiate daily movement of hands and elbows from the first postoperative period.

c. Scapular exercises, with sling, after 2 weeks postoperatively.

d. Pendulum exercises after removal of sling.

e. After 1-2 weeks exercises to gain range of motion and up to 3 months to avoid exercises in zone of instability (maximum external rotation, abduction and extension of the shoulder).

f. After 3 months, from the date of surgery, exercises to gain strength of the rotator cuff and scapular girdle, starting with isometric and after active.

g. Return to sports activities, depending on the degree of contact, in 6-8 months.

\section{Results}

Fifty-six patients were operated with $67.8 \%(n=38)$ by the arthroscopic technique and $32.2 \%(n=18)$ by the open technique. The results obtained show a predominance of Bankart lesion in males $87.5 \%(n=49)$ in relation to females $12.5 \%(n=7)$ (Table 1-3). Failure was observed in $9.2 \%(n=3)$ of the cases operated by the arthroscopic technique and $0 \%(n=0)$ in which the open technique was performed. The correlation of the mean with the clinical and functional results demonstrates advantage of the arthroscopic technique in the stability and mobility criteria. The open technique demonstrates advantage in the criteria: less pain, function and final Carter-Rowe functional outcome.

Table 1: Epidemiological analysis of sex and surgical techniques.

\begin{tabular}{|c|c|c|c|}
\hline & Men & Women & Total \\
\hline \multirow{2}{*}{ Arthroscopic } & 3.4 & 4 & 38 \\
& $-60.70 \%$ & $-7.10 \%$ & $-67.80 \%$ \\
\hline \multirow{2}{*}{ open } & 15 & 3 & 18 \\
& $-26.70 \%$ & $-5.40 \%$ & $-32.20 \%$ \\
\hline \multirow{2}{*}{ Total } & 49 & 7 & 56 \\
& $-87.50 \%$ & $-12.50 \%$ & $-100 \%$ \\
\hline
\end{tabular}

Table 2: Epidemiological analysis of the first dislocation and surgical techniques.

\begin{tabular}{|c|c|c|c|}
\hline & Arthroscopic & Open & Total \\
\hline Less than 20 years & $\begin{array}{c}6 \\
(-10.70 \%)\end{array}$ & $\begin{array}{c}3 \\
(-5.40 \%)\end{array}$ & $\begin{array}{c}9 \\
(-16.10 \%)\end{array}$ \\
\hline 20 to 25 years & $\begin{array}{c}15 \\
(-26.70 \%)\end{array}$ & $\begin{array}{c}4 \\
(-7.10 \%)\end{array}$ & $\begin{array}{c}19 \\
(-33.90 \%)\end{array}$ \\
\hline 25 to 30 Years & $\begin{array}{c}9 \\
(-16.10 \%)\end{array}$ & $\begin{array}{c}4 \\
(-7.10 \%)\end{array}$ & $\begin{array}{c}13 \\
(-23.20 \%)\end{array}$ \\
\hline $30-40$ years & $\begin{array}{c}5 \\
(-8.90 \%)\end{array}$ & $\begin{array}{c}3 \\
(-5.40 \%)\end{array}$ & $\begin{array}{c}8 \\
(-14.20 \%)\end{array}$ \\
\hline $40-50$ years & $\begin{array}{c}2 \\
(-3.60 \%) \\
\end{array}$ & $\begin{array}{c}4 \\
(-7.10 \%) \\
\end{array}$ & $\begin{array}{c}6 \\
(-10.70 \%) \\
\end{array}$ \\
\hline More than 50 years & $\begin{array}{c}1 \\
(-1.70 \%)\end{array}$ & $\begin{array}{c}1 \\
(-1.70 \%)\end{array}$ & $\begin{array}{c}2 \\
(-3.40 \%)\end{array}$ \\
\hline Total & $\begin{array}{c}38 \\
(-67.80 \%)\end{array}$ & $\begin{array}{c}18 \\
(-32.10 \%)\end{array}$ & $\begin{array}{c}56 \\
(-100 \%)\end{array}$ \\
\hline
\end{tabular}

Table 3: Comparison between affected side and average number of dislocations in which the patient seeks orthopedic assistance.

\begin{tabular}{|c|c|c|c|}
\hline & $\begin{array}{c}\text { Dominant } \\
\text { Side Affected }\end{array}$ & $\begin{array}{l}\text { Nodominant } \\
\text { Side Affected }\end{array}$ & Total \\
\hline 1 to 3 Dislocations & $\begin{array}{c}13 \\
(-23.20 \%)\end{array}$ & $\begin{array}{c}3 \\
(-5.40 \%)\end{array}$ & $\begin{array}{c}16 \\
(-28.60 \%)\end{array}$ \\
\hline 4 to 10 Dislocations & $\begin{array}{c}10 \\
(-17.80 \%)\end{array}$ & $\begin{array}{c}9 \\
(-16.10 \%)\end{array}$ & $\begin{array}{c}19 \\
(-33.90 \%)\end{array}$ \\
\hline $\begin{array}{c}11 \text { to } 20 \\
\text { Dislocations }\end{array}$ & $\begin{array}{c}5 \\
(-8.90 \%)\end{array}$ & $\begin{array}{c}3 \\
(-5.40 \%)\end{array}$ & $\begin{array}{c}8 \\
(-14.30 \%)\end{array}$ \\
\hline $\begin{array}{c}\text { More than } 20 \\
\text { Dislocations }\end{array}$ & $\begin{array}{c}5 \\
(-8.90 \%)\end{array}$ & $\begin{array}{c}8 \\
-14.30 \%\end{array}$ & $\begin{array}{c}13 \\
-23.20 \%\end{array}$ \\
\hline Total & $\begin{array}{c}33 \\
(-59 \%)\end{array}$ & $\begin{array}{c}23 \\
(-41 \%)\end{array}$ & $\begin{array}{c}56 \\
(-100 \%)\end{array}$ \\
\hline
\end{tabular}


There is divergence in the literature when the failure rate in the procedures. They cite failure of the arthroscopic procedure: Barnes et al 6\%, Boileau et al 26\%, Ryu et al 27\%.When open technique, Rowe et al., 8\%, Hawkins et al., Levine et al., 20\% and Walch et al. These works do not compare techniques [8-15]. In this study, the result of failure was $7.9 \%$ in the arthroscopic technique and $0 \%$ in the open technique.

The work of Fabbriciani [16] and cols, 2006, comparing 30 Patients operated by open technical with 30 operated arthroscopic technique peels, brought result with level I evidence in BOTH techniques them in terms of stability provide or even as result Stability However the open Technique could compromise the recovery of the full range of shoulder movement. In this work the results are in agreement with the one described in the literature since the stability criterion showed no difference between the techniques (Table 4-6).

Table 4: Correlation of surgical failure and surgical techniques.

\begin{tabular}{|c|c|c|c|}
\hline & Initial n & $\mathrm{N}$ end & Failure \\
\hline \multirow{2}{*}{ Arthroscopic } & \multirow{2}{*}{38} & \multirow{2}{*}{35} & 3 \\
\hline & & & $(-9.20 \%)$ \\
\hline \multirow{2}{*}{ Open } & \multirow{2}{*}{18} & \multirow{2}{*}{18} & 0 \\
\hline & & & $(0 \%)$ \\
\hline
\end{tabular}

Table 5: Correlation of mean and standard deviation with clinical results and functional score.

\begin{tabular}{|c|c|c|}
\hline & $\begin{array}{c}\text { Standard Deviation } \\
\text { Arthroscopic }\end{array}$ & $\begin{array}{c}\text { Standard Deviation } \\
\text { Open }\end{array}$ \\
\hline Pain & $7.85 \pm 2.78$ & $8: 33 \pm 2: 42$ \\
\hline Anointing F & $45.28 \pm 7.94$ & $45.83 \pm 6.91$ \\
\hline And stability & $28.71 \pm 5.60$ & $28.33 \pm 4.85$ \\
\hline Mobility & $7.28 \pm 2.80$ & $7: 22 \pm 3: 07$ \\
\hline Rowe & $89.14 \pm 1.14$ & $11: 56 \pm 89.72$ \\
\hline
\end{tabular}

Table 6: T-test correlation for independent groups, significant analysis and interpretation.

\begin{tabular}{|c|c|c|c|}
\hline & $\mathbf{T}$ & $\mathbf{P}$ & Interprets CaO \\
\hline Dor (videoversus Open) & -0.614 & 0.541 & Ns \\
\hline $\begin{array}{c}\text { anointingF (videoversus } \\
\text { Open) }\end{array}$ & -0.247 & 0,805 & Ns \\
\hline $\begin{array}{c}\text { And stability (videoversus } \\
\text { Open) }\end{array}$ & 0.244 & 0.807 & Ns \\
\hline Mobility (videoversus Open) & 0.0755 & 0.94 & $* * *$ \\
\hline Rowe (videoversus Open) & $-0,150$ & 0.88 & Ns \\
\hline
\end{tabular}

Regarding the mobility criterion, the patients operated by arthroscopic presented better results. The work of Mohtadi [16] JBJS - 2014 with a Level I Sample evidence how we recurrence brainy $11 \%$ and $23 \%$ open procedures That We arthroscopic procedures and Prove how brainy or functional outcome no statistical significance. In this study, the relapse in the cases operated by arthroscopy was also superior to the open surgery and the final functional result was not statistically significant difference between both techniques (Figure 1\&2).

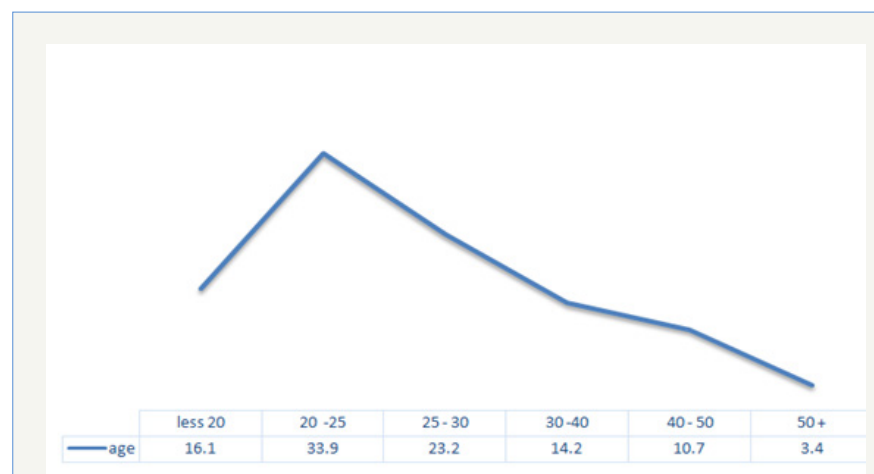

Figure 1: Epidemiological distribution according to age group.

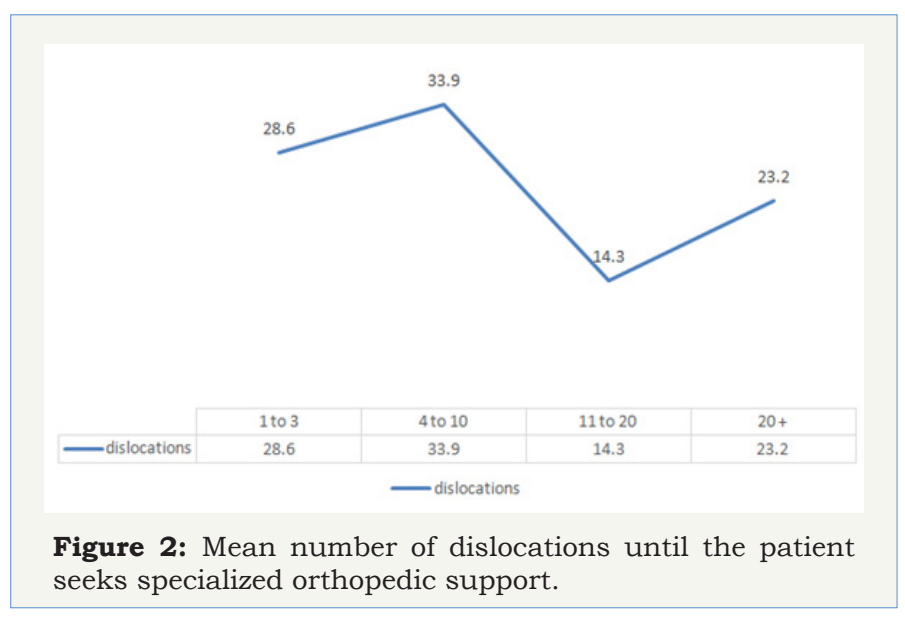

\section{Conclusion}

It is possible to conclude with this work that:

a. Both techniques present good results, although few works in the literature compare homogeneous groups of patients.

b. The possibility of further episodes of dislocation is apparently greater in the arthroscopic technique.

c. The arthroscopic technique seems to have a better result when compared to mobility.

\section{References}

1. Streubel PN, Krych AJ, Simone JP, Dahm DL, Sperling JW, et al. (2014) Anterior glenohumeral instability: a pathology-based surgical treatment strategy. J Am Acad Orthop Surg 22(5): 283-294.

2. Burks RT, Presson AP, Weng HY (2014) An analysis of the technical aspects of the arthroscopic Bankart Procedure Performed the in the United States. Arthroscopy 30(10): 1246-1253.

3. Lippitt S, Matsen F (1993) Mechanisms of Glenohumeral Joint stability. Clin Orthop Relat Res 291: 20-28.

4. PerthesG (1906) Über Operationen Bei Habitueller Schulterluxation. DtschZ Chir. 
5. Bankart ASB (1939) The pathology and treatment of recurrent dislocation of the shoulder joint. 26(101): 23-29.

6. Zarins B (1993) Previous shoulder stabilization using the Bankart procedure, Arthorscopy Sports Med Rev 1: 259.

7. Godinho GG, et al. (2014) Procedure Arthrosis or Bankart peak: Comparative study of the use wired The Anchors double simple or Ap or Following two years. Rev Bras Ortop.

8. Chen L, Xu Z, Peng J, Xing F, Wang H, et al. (2015) Effectiveness and safety of Arthroscopic Versus Open Bankart Repair for recurrent anterior shoulder dislocation: a meta-analysis of clinical trial date. Arch Orthop Trauma Surg 135(4): 529-538.

9. Ng C, Bialocerkowski A, Hinman R (2007) Effectiveness of Arthroscopic Versus Open Surgical Stabilization For the management of traumatic Glenohumeral Instability. Int J Evid Based Healthc 5(2): 182-207.

10. Mohtadi NG, Bite IJ, Sasyniuk TM, Hollinshead RM, Harper WP (2005) Arthroscopic Versus Open Repair for traumatic anterior shoulder instability: the meta-analysis. Arthroscopy 21(6): 652-658.

11. TR lenses, Franta AK, WolfFM, Leopold SS, Matsen FA (2007) Arthroscopic compared with open repairs for recurrent anterior shoulder instability. A systematic review and meta-analysis of the literature. J Bone Joint Surg Am 89(2): 244-254.
12. Mazzocca AD, Brown FM, Career DS, Hayden J, Romeo AA (2004) Open instability repairs: still the gold standard? In: Orthopedics Today NY CME Course. Orthopedi 14. 54.

13. Armstrong A, Boyer D, Ditsios K, Yamaguchi K (2004) Arthroscopic versus open treatment of anterior shoulder instability. Instr Course Lect 53: 559-563.

14. Kim SH, Ha KI, Kim SH (2002) Bankart Repair In traumatic anterior shoulder: Instability: Open Versus Arthroscopic Technique. Arthroscopy 18(7): 755-763.

15. Fabbriciani C, Milano G, Demontis A, Fadda S, Ziranu F, et al. (2004) Arthroscopic Versus Open Treatment of Bankart Lesion of the shoulder: a prospective randomized study. Arthroscopy 20(5): 456-462.

16. Dahm DL (2014) Is open stabilization superior to Arthroscopic Stabilization for the Treatment of Recurrent Traumatic Shoulder Instability Previous? Commentary on an article. In: Nicholas GH Mohtadi MD (Eds.), A Randomized Clinical Trial Comparing Open and Arthroscopic Stability for Recurrent Traumatic Shoulder Instability Previous: two-Year Follow-up With Disease-specific quality-of-life outcomes. J Bone Joint Surg Am 96(5): e41. 